\title{
Contents, Vol. 76, 1932
}

Inhaltsverzeichnis.

Eigenarbeiten. sen $<$

Bartels, M., Das Krankheitsbild des Augenzitterns der Bergleute in England und Deutscbland und sein Ein-

fluß auf die Erwerbsfähigkeit 29

Boenheim, F., Die Leberfunktion bei Laurence-Biedlscher

Krankheit 156

Blatt, N,, „Replantatio Pterygii” als Methode der Flügel-

felloperation $16 \mathrm{r}$

v. Csapody-Mócsy < Handarbeit und Sehschärfe .... 287 Fialho, A., Beiträge und Erläuterungen zur Sklerektomie.

Behandlung des chronischen Glaukoms 346

Hanssen, R., Beitrag zur pathologischen Anatomie der

Linse (Cataracta pulverulenta) 73

,-- , Beitrag zur pathologischen Anatomie des Glas-

körpers (Synchisis scintillans) 77

Jordan, S., Ein Beitrag zur Bindehauttuberkulose . . 147 Junius, P., Krankheiten durch Störung des Lipoidstoff-wechsels (Niemann-Picksche Krankheit, Morbus Gaucher, Schüller-

Christiansche Krankheit, Xantho-matosen verschiedener Lokalisation) in Beziehungen zum

Sehorgan. Eine Zusammenfassung .... I2c >

,-- , Familiäre amaurotische Idiotie 225

Kestenbaum, A., Zur topischenDiagnostik derHemianopsie 241

Krassó, I., Über Lenticonus posterior 278

Kreibig, W., Über leukämische Fundusveränderungen . 358 Kunz, E., Zur Trachomstatistik in Ostpreußen .... 293 Landegger, G., Über einen Fall von schwerer sulziger

Skleritis 83

Lange, C, Über moderne Luesdiagnostik mit besonderer

Berücksichtigung des Liquor cerebrospinalis ... 1 Lauber, H., Über optisch wirksame

Sicherheitsgläser . . 238 Metier, /., Über die Schaffung von fiächenhaften Ver-lötungen

derNetzhaut mit der Aderhaut durch Endo-thermie. Nachtrag zu der Arbeit in Band 75, Heft 490

Procksch, M., Über die Behandlung von Tränensackphleg-monen und akuten Schwellungen

derLidermit Kokain-

Einlagen in die Nase 283

Reitsch, W., Supercilium und Pars orbitalis palp. sup. und

ihre Bedeutung für das individuelle Auge .... 61 Safáf, K., und W. Spitzmüller,

Elektrokoagulation als neues Behandlungsverfahren gegen schweren Blepharo-

spasmus .. 337

Spitzmüller, W., siehe Safáf, K.

Wiesli, P., Perkain als Oberflächenanästhetikum in der 
Augenheilkunde

157

- IV -

Seite

Aus der Praxis îur die Praxis.

Handmann, M., Spontane Verdünnung der Lederhaui. als

Störung bei Staroperation 365

Ohm, /., Zur Frage der Bindehautdeckung bei der Star-operation ... . . . ‘. ‘. “.. . . . . . . . 300

Berichte über die opMhalmologiscl $><\hat{1}$ Literatur.

Normale und pathologische Anátomie. Von Priv.-Doz.

Dr. Schneider in Graz . . . . . . . . . . . 166

Entwicklungsgeschichte des Auges. Mißbildungen. An-

thropologie. Von Prof. Or., R. Seefelder in Innsbruck 178 Geschichte der Ophthalmologie.

sVГon Prof. Dr. R, Seefelder

in Innsbruck . . . . . ."“,' . ... . .... 194

Physiologische Optik. Von T > x.i'B, Daniels in Hamburg 303 Psychologische Öptik. Von Or.

Ê. Daniels in Hamburg 318 Sensibilität, vom Auge ausgelöste Rèflexe. Von Dr.

B. Daniels in Hamburg ...' ... . . . . . . 321

Vererbung und Auge. Von Dr. A. Franc'eschetti in Basel 386 Physikalische Optik, Dioptrik des Auges, Methoden der

Refraktionsbestimmung. Von Dr. R. Kramer in Wien 369 Klinik und Entstehung der

Refraktionsanomaíien. Seh-

schärfe. Von Dr. K. Safâr und Dr. L. Sallmann in , Wien . . . . . . . . . . . , , . . . 377

Gesellschaîtsberichte.

Berliner Augenärztliche Gesellschaft. Sitzu,ngvom 23. April 1931325

Sitzung vom 4. Juni 1931326

Sitzung vom22. Oktober 193190

Sitzung vom 26. Novbr. 1931198

Ophthalmolog. Gesellschaft in Wien. Sitzung vom.19. Oktober 193194

Sitzungvom 11. Novbr. 1931200

Sitzung vom 14. Dezbr. 1931403

Sitzung vom 18. Januar 1932 40535. Tagung der Vereinigung mitteldeutscher Augenärzte am 5. und

6. Sept. T931 in Jena 206

$\lambda^{\wedge}$ erein der Augenärzte von Ost- und Westpreußen. Sitzung vom

13. Juni $1931 \quad 328$

TJngarische Ophthalmologische Gesellschaft in Budapest. Jahres-

versammlung am 6. und 7. Juni 1931 . 96

Sitzung am 30. Oktober 1931 408Gemeinsame Sitzung des Ungarischen Vereins fur RheumaFor-schung und der Ungarischen Ophthalmologischen Gesellschaft

am 5. Juni 1931

Diagnose und Therapie $\quad 120,221,330,412$

Unfall- und Versicherungskunde $\quad .122,334$

Buchbesprechungen ... 124, 335, 416

Personalien 125, 336

Tagesnachricliten $\quad 127,224,417$

Berichtigungen 224, 336 
S. Saehregister zu Band $76 \quad 420$

jVamenregister zu Band $76 \quad 426$ 